\title{
Las emociones: necesidad de su programación para una actividad física más saludable
}

\section{Pedro Sáenz-López Buñuel}

\section{Universidad de Huelva Email: psaenz@uhu.es}

Este artículo fue publicado como capítulo del libro: Sáenz-López, P. (2015). Las emociones: necesidad de su programación para una actividad física más saludable. En Moreno-Murcia, J. A. y De Souza, M. C. (coord.). Motricidad humana. Hacia una vida más saludable (pp 107-128). Santiago: Universidad Central de Chile.

\section{RESUMEN:}

El concepto de salud ha evolucionado desde una concepción prioritariamente curativa, es decir, ausencia de enfermedad, a un planteamiento más preventivo. Sin embargo, la propia definición de la Organización Mundial de la Salud va más allá y plantea como meta el bienestar. Este es el reto de la sociedad del siglo XXI. Las evidencias científicas aportan cada vez más datos sobre la influencia que la gestión emocional y la actividad física tienen sobre el bienestar personal y social. Por tanto, los objetivos que nos planteamos fueron analizar la relación entre estos dos tópicos, describir la influencia que tienen sobre la salud y el bienestar y realizar recomendaciones para una actividad física emocionante y saludable. Entre las conclusiones, destacamos la influencia que el ejercicio físico produce en las emociones ya que genera hormonas responsables del bienestar o del aprendizaje cognitivo por lo que ayuda a tener más energía, más autoestima, más autocontrol, menos estrés, mejora la toma de decisiones inteligente, las relaciones sociales, facilita aprendizajes, etc. Por su parte, la inteligencia emocional facilita una práctica deportiva más eficiente y satisfactoria. Entre las recomendaciones, se aportan ideas como plantearse objetivos claros y a corto plazo, establecer una comunicación positiva con nosotros mismos y con los demás, vivir cada experiencia como un éxito, rodearse de amigos activos y saludables y sobre todo disfrutar de la práctica de actividad física antes, durante y después de su realización.

PALABRAS CLAVE: inteligencia emocional, ejercicio físico y bienestar. 


\section{Emotions: the need to program them for a healthier physical activity}

\section{ABSTRACT:}

The concept of health has evolved from a primarily curative conception, that is, absence of disease, a more preventive approach. However, the definition of the World Health Organization goes further and sets welfare as a goal. This is the challenge of the society of the 21 st century. Scientific evidence provides more and more data on the influence that emotional management and physical activity have on personal and social well-being. Therefore, the objectives we set were to analyze the relationship between these two topics, to describe the influence they have on health and well-being and to make recommendations for an exciting and healthy physical activity. Among the conclusions, we highlight the influence that physical exercise produces on emotions as it produces hormones responsible for well-being or cognitive learning, so it helps to have more energy, more self-esteem, more self-control, less stress, improves intelligent decision making, social relations, facilitates learning, etc. On the other hand, emotional intelligence facilitates a more efficient and satisfactory sports practice. Among the recommendations, there are ideas such as setting clear and short goals, establishing positive communication with ourselves and others, living each experience as a success, surrounding yourself with active and healthy friends, and especially enjoying the practice of physical activity before, during and after its completion.

KEY WORDS: emotional intelligence, physical exercise and well-being. 


\section{INTRODUCCIÓN}

\section{1. ¿Por qué abordar esta temática?}

La inteligencia emocional y la Actividad Físico-deportiva van a ser protagonistas en ámbitos tan trascendentes como la educación y la salud del siglo XXI. Cada vez más investigadores, especialmente neurocientíficos, aportan datos concluyentes a este respecto. Por ejemplo, Goleman (1996), a través de las evidencias de numerosos estudios, afirma que entre el 60 y $80 \%$ de las enfermedades actuales son de origen psicosomático. Los avances médicos están permitiendo que los seres humanos aumenten su esperanza de vida. Sin embargo, el envejecimiento de la población va a conllevar que en los próximos años se tripliquen los casos de Alzehimer (Bilbao, 2013). Este psicólogo, experto en enfermedades neurodegenerativas, propone cómo cuidar el cerebro para prevenir o retrasar la aparición de Alzehimer, Ictus o Parkinson. De los seis factores que más evidencias científicas han mostrado para prevenir estas enfermedades, tres están relacionadas con la inteligencia emocional (alejar el estrés, cultivar emociones positivas y una vida social satisfactoria). El ejercicio físico, la alimentación y la reserva cognitiva completan los factores claves, a día de hoy, para cuidar el cerebro, según este autor.

A pesar de las evidencias científicas sobre los beneficios de la Actividad Físicodeportiva sobre la salud, los niveles de sedentarismo son inquietantes (Instituto Nacional de Estadística, 2013). A modo de ejemplo, en algunas comunidades autónomas cerca del $80 \%$ de la población está fuera de los parámetros mínimos de actividad física recomendados para la salud.

El estilo de vida de la sociedad occidental ha sido denominado como la sociedad del bienestar debido al aumento exagerado de comodidades (transportes, ascensores, mandos a distancia, escaleras mecánicas, ...). Paradójicamente, este estilo de vida tiene una influencia directa en el aumento de obesidad, colesterol o enfermedades cardiacas, cuya relación con la salud y la calidad de vida es evidente. Además, como afirman Fernández-Berrocal y Extremera (2010), las enfermedades mentales están aumentando a un ritmo inquietante. Por tanto, el estilo de vida actual se podría denominar de pseudo-bienestar ya que las comodidades y el sedentarismo son causa de números problemas relacionados con la salud. Punset Casals (2010) recuerda que los seres humanos han casi triplicado la esperanza de vida en un periodo evolutivo muy corto (poco más de 200 años). Este hecho sin precedentes en la evolución, ha supuesto que nuestro cerebro esté falto de recursos para gestionar este tiempo y esta situación. Quizás por esta razón, afirma este autor, tenemos una capacidad infinita para ser infelices. El estilo de vida actual ayuda poco al objetivo de sentirse bien, es decir a la búsqueda del bienestar. Paralelamente, Bizquerra (2000) ofrece datos sobre la prevalencia de comportamientos problemáticos como la violencia, la depresión, el consumo de tabaco, alcohol o drogas, anorexia, obesidad, etc. que justifican sobradamente la necesidad de una adecuada educación emocional. En el siglo XX, aprendimos a sobrevivir físicamente, por fuera, sin embargo, en el siglo XXI ante la avalancha de enfermedades mentales y de origen emocional, necesitamos aprender a sobrevivir por dentro, emocionalmente (Punset Bannel, 2012). A lo largo del capítulo se podrá comprobar cómo la actividad física ayuda al desarrollo de la inteligencia emocional y al bienestar.

Por todo lo dicho, merece la pena analizar la relación de dos de los factores que más evidencias científicas han mostrado en su influencia sobre la salud y, especialmente, en la búsqueda del auténtico bienestar personal y social: la actividad físico-deportiva y la gestión de las emociones. 


\subsection{Objetivos}

Los objetivos de este artículo se concretan en:

- Analizar la inteligencia emocional y su relación con la actividad físico-deportiva.

- Valorar la importancia de la actividad física y la inteligencia emocional en la salud preventiva y su influencia en el bienestar.

- Realizar propuestas para desarrollar una actividad física emocionante y saludable en busca del bienestar personal y social.

\section{LA SALUD DEL SIGLO XXI: EL BIENESTAR PERSONAL Y SOCIAL}

\subsection{Evolución del concepto de salud}

La Organización Mundial de la Salud -OMS- (2010) define la salud como un estado de completo bienestar físico, mental y social, y no solamente la ausencia de afecciones o enfermedades. A pesar de que esta definición tiene más de 50 años, el sistema sanitario se ha centrado principalmente en el concepto de intervenir cuando hay un problema. Esta Medicina curativa, ha sido predominante, motivo por el cual la OMS indicó en la definición que la Salud es mucho más que la ausencia de enfermedad. La gente ha acudido al médico cuando se encontraba mal y los avances científicos se han centrado principalmente en cómo curar enfermedades. En las últimas décadas, ha aumentado la atención a la salud preventiva (Sociedad española de medicina preventiva, salud pública e higiene, 2014). Las vacunas o el programa de "control del niño sano" son dos ejemplos de medicina preventiva cuyo foco está puesto en evitar la enfermedad. El gran reto de la salud del siglo XXI es aumentar el bienestar personal y social de la población, a pesar de que éste sea el centro de la definición de la OMS de hace más de 50 años. Los datos que hemos dado en la introducción muestran que los sistemas sanitario y educativo trabajan todavía alejados de este objetivo. A continuación, se describirán evidencias de la influencia de las emociones en la salud en los tres niveles, tanto el curativo, como el preventivo y principalmente en la búsqueda del bienestar. 


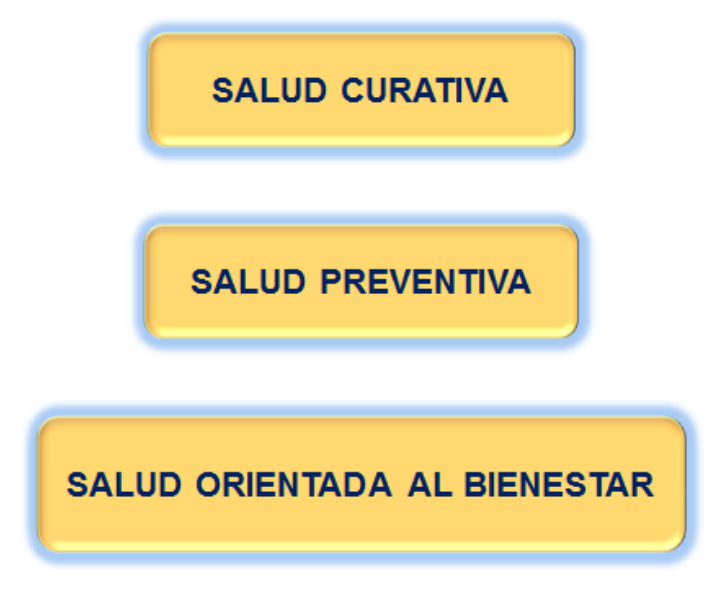

Figura 1. Evolución del concepto de salud

\subsection{Relación de las emociones con la salud}

En la web del Colegio Oficial de Psicólogos de Madrid (2014) a través del Proyecto "emociones y salud" se describe la influencia que tiene la forma en que reaccionamos a los problemas y estresores cotidianos como el dinero, pareja, hijos, abuelos, trabajo, exámenes, etc. en la salud. Nuestras respuestas al estrés se pueden dar en forma de discusiones, enfados, agobios, ansiedad, miedos o tristezas. Estas respuestas emocionales al estrés suelen ser la causa de alteraciones y enfermedades como resfriados, herpes, alergias, dolores de cabeza o espalda, problemas intestinales o cardiacos. Además, la mayoría de los hábitos perjudiciales para la salud como fumar, beber, tomar drogas o el sedentarismo, están relacionados con estas respuestas inapropiadas al estrés. Limonero, Tomás-Sábado, y Fernández-Castro (2006) demuestran cómo niveles altos inteligencia emocional, especialmente de regulación emocional, correlacionan con menos consumo de tabaco y cannabis. La mayoría de estos hábitos dañinos se generan al tratar de compensar déficits emocionales por falta de otros recursos. La realidad es que cada persona reacciona de forma diferente a cada situación en función de cómo ha aprendido, principalmente durante su infancia (Punset Casals, 2010). Numerosas investigaciones demuestran que lo que produce estos problemas no son las situaciones estresantes, sino cómo se valoran y se afrontan. Como afirma Sapolsky (1994), el ser humano es el único animal capaz de generar hormonas de estrés gracias a nuestra poderosa, aunque mal-educada y mal-entrenada imaginación. En su libro "por qué las cebras no tienen úlcera" explica, para entender esta idea, que la cebra sólo se estresa cuando es perseguida por un león. Cuando éste caza una presa, el resto de herbívoros sigue pastando tranquilamente a unos pocos metros del carnívoro comiendo, porque tiene la certeza de que el peligro ya ha pasado. El ser humano se estresa ante numerosas situaciones (enfados, miedos, agobios, situaciones tristes...) tanto cuando ocurre el hecho como posteriormente, pensando o recreándose en el acontecimiento, hablando con sus familiares y amigos, etc. Cada conversación o cada vez que ese hecho ocupa nuestro pensamiento, generamos las mismas hormonas defensivas y estresantes que en el momento que se produjo.

Esta influencia de los estados emocionales sobre la salud ha sido ratificada por la ciencia a través de estudios como los de Felten y Cohen (2006) quienes demostraron que las emociones tienen un efecto muy poderoso sobre el sistema nervioso autónomo y el sistema inmunológico. Esto explica las evidencias científicas que hay tanto en la salud curativa, como preventiva y, definitivamente en la búsqueda del bienestar. 
Es evidente que la mayoría de las enfermedades mentales se curan o mejoran con una reeducación emocional a través de un adecuado proceso terapéutico (Colegio Oficial de Psicólogos de Madrid, 2014). Lo que sorprende, son las evidencias sobre la influencia que las emociones positivas ejercen sobre el curso de cualquier enfermedad. Por ejemplo, Goleman (1996) cita diversos estudios que han mostrado cómo las personas optimistas se recuperan antes y mejor que las pesimistas de una enfermedad. También se ha demostrado también los efectos negativos de la depresión en la curación de enfermedades e incluso en ser causa de algunas (Davidson, 2012).

La gestión de las emociones es también uno de los más potentes factores de la medicina preventiva. A modo de ejemplo, Goleman (1996) cita un estudio de Sheldon Cohen quien expuso a sujetos con diferentes niveles de estrés al virus del resfriado y enfermaron el doble de sujetos con alto nivel de estrés respecto a los que tenían bajos niveles. Otro potente factor emocional preventivo son las relaciones, ya que se ha demostrado que las relaciones sociales satisfactorias reducen el estrés. Rodearse de gente, de amigos, de familiares, mostrar cariño y sentirse querido supone un factor preventivo de enfermedades al mismo nivel que evitar fumar, hacer ejercicio físico o tener un peso adecuado (Goleman, 1996). Esto es particularmente importante en las relaciones con las personas con las que vivimos cotidianamente cuya incidencia sobre la salud correlaciona con el clima de tranquilidad o de estrés en el que se vive (Cacioppo y Patrick, 2008).

Respecto a la influencia perjudicial de las emociones negativas sobre la salud, las evidencias son aún mayores. Las emociones tóxicas, especialmente la ira, la ansiedad y la depresión, pueden ser tan perjudiciales para la salud como fumar varios paquetes de tabaco al día o como tener el colesterol alto (Piqueras, Ramos, Martínez, Oblitas, 2009). El estrés, a través de hormonas como el cortisol, la adrenalina o la prolactina disminuye la resistencia inmunológica. Por ejemplo, la ira muestra unos efectos nocivos directos sobre el corazón. Nasser (2010) cita una serie de investigaciones que demuestran la alta correlación entre las personas que se irritan con facilidad respecto al aumento de enfermedades cardiacas. En el hombre se desarrolla más la ira mientras en la mujer la ansiedad y el miedo cuyos efectos negativos sobre la salud han sido también demostrados por diversos estudios (Goleman, 1996). El enfado es una emoción necesaria, como todas, pues reprimirlo también puede tener consecuencias negativas. Lo recomendable es evitar el estado constante de mal humor o de emociones negativas y aprender a identificarlas y gestionarlas con utilidad.

Si las emociones son importantes en la salud curativa y en la salud preventiva, en el reto del siglo XXI, que es el bienestar personal y social, la gestión útil e inteligente de nuestros estados emocionales es la clave. El bienestar, en líneas generales, se corresponde con la alegría, satisfacción con la vida que deriva de un funcionamiento óptimo (McDowell, 2010), en el que las emociones positivas (Diener, 1994) y la autoestima (Gagné, Ryan y Bargmann, 2003) aparecen como indicadores fiables. El bienestar es un constructo que describe el estado en el que se encuentra la vida de la persona (McGillivray, 2007). A pesar de esta sencilla definición, se trata de un concepto complejo que va más allá de reducir las situaciones que produzcan experiencias negativas y aumentar las positivas (Ryan y Deci, 2001). Como comenta Punset Casals (2010), nadie nos ha enseñado a controlar nuestros pensamientos a pesar de que numerosos autores han demostrado que es posible. La inteligencia emocional desarrolla una serie de competencias como el control de los pensamientos y emociones, el autoconocimiento, la capacidad de auto-motivarse, de retrasar una gratificación o la habilidad de empatizar y confiar en los demás (Goleman, 1996). El concepto de 
inteligencia emocional y su relación con la salud y principalmente con el bienestar van a ser desarrollados a continuación.

\section{LA INTELIGENCIA EMOCIONAL Y EL BIENESTAR PSICOLÓGICO}

\subsection{Las emociones}

Las emociones son el resultado de cómo experimentamos física y mentalmente la interacción entre nuestro mundo interno y externo. Se expresan a través de comportamientos, expresiones de sentimiento y cambios fisiológicos.

Las emociones son naturales y tienen o han tenido su utilidad. Es necesario reconocerlas, aceptarlas y utilizarlas en beneficio de nuestro bien-estar. Autores como Damasio (2001) demuestran que incluso son imprescindibles para tomar buenas decisiones, al contrario de lo que hasta ahora se creía. El Colegio Oficial de Psicólogos de Madrid (2014) confirma que todas las emociones son útiles, incluso las llamadas negativas. El miedo nos salva la vida a diario, la ira nos da dignidad o la tristeza nos ayuda a reflexionar, relativizar y crecer. El problema es cuando estas emociones son excesivamente intensas o duraderas pues, como se ha expuesto anteriormente, nos hacen más vulnerables a contraer enfermedades, nos dificulta la curación y nos alejan del bienestar.

Para comprender la dimensión que puede abarcar la inteligencia emocional, resulta interesante distinguir, siguiendo a Davidson (2012), los diferentes niveles emocionales. La unidad más pequeña es el estado emocional cuya duración es de unos segundos y suele desencadenarse como reacción a una experiencia, una noticia o un pensamiento. Si este sentimiento persiste minutos, horas o días se convierte en un estado de ánimo. Si la duración de este estado es de años se llama rasgo emocional. Este autor afirma que cada persona desarrolla un perfil emocional en base a cómo ha aprendido a responder emocionalmente a las experiencias vividas. La utilidad y eficiencia en el manejo de competencias emocionales en busca del bienestar personal y social es lo que Goleman (1996) denominó inteligencia emocional.

\subsection{Consecuencias saludables al mejorar la inteligencia emocional}

En las últimas décadas, ha crecido el interés del estudio por asociar la inteligencia emocional con los comportamientos referidos a la salud (Austin, Saklofskeb, y Eganc, 2005), proponiendo una nueva perspectiva en el estudio de las emociones (Extremera y Fernández-Berrocal, 2006). En este sentido, diversas investigaciones han vinculado el bienestar con la inteligencia emocional (Extremera, Ruiz-Aranda, Pineda, y Salguero, 2011; Rey, Extremera y Pena, 2011).

El desarrollo del concepto de inteligencia emocional arranca de los estudios de Salovey y Mayer (1990), representado a ésta como parte de la inteligencia social, lo que sugiere que ambos conceptos están relacionados y pueden, con toda probabilidad, presentar componentes interrelacionados de la misma construcción (Bar-On, 2006). En esta línea, autores como Fernández-Berrocal, Ramos, y Extremera (2001) consideran que la inteligencia emocional presenta gran importancia en cuanto a la adaptación a diferentes contextos. Por ejemplo, el desarrollo de relaciones interpersonales positivas se asocia a cierto grado de desarrollo de inteligencia emocional (Schutte et al., 2001). Así, Lopes, Salovey, y Straus (2003) indicaron la relación existente entre la inteligencia emocional y la calidad de las relaciones sociales, dado que un uso inteligente de las 
emociones es esencial para la propia adaptación física y psicológica al contexto social (Mayer y Salovey, 1990; Salovey, Mayer, Goldman, Turvey, y Palfai, 1995).

Anteriormente, Gardner (2001) había expuesto dentro de la teoría de las inteligencias múltiples, que la inteligencia emocional incluye la inteligencia intrapersonal y la inteligencia interpersonal o social. Este autor argumenta la necesidad de desarrollar estas inteligencias ya que son útiles para toda la población. Su inclusión en el sistema educativo es una necesidad. En esta línea, Extremera y Fernández-Berrocal (2004) encontraron cuatro problemas asociados a bajos niveles de inteligencia emocional en el contexto educativo: déficit en los niveles de bienestar y ajuste psicológico del alumnado, disminución en la cantidad y calidad de las relaciones interpersonales, descenso del rendimiento académico y aparición de conductas disruptivas y consumo de sustancias adictivas. La inteligencia emocional es, por tanto, un importante indicador de éxito en muchos aspectos de la vida (Bar-On 2002; Goleman, 1996). Ciarrochi, Chan y Caputi (2000) también la han asociado positivamente, con variables de la personalidad, como la autoestima.

Gran parte de la investigación en esta temática se ha interesado en el estudio de las habilidades emocionales como predictoras del bienestar psicológico, salud y relaciones interpersonales (Heck y Oudsten, 2008), debido a que las respuestas psicofisiológicas al estrés pueden ser un mecanismo subyacente del funcionamiento emocional (Salovey, Stroud, Woolery, y Epel, 2002). Desarrollando una inteligencia emocional adecuada, es posible mejorar la gestión de las emociones, tomar decisiones más saludables y más eficaces cuando existen situaciones de estrés y estados de ánimo negativos (Damasio, 2001; Meyer y Zizzi, 2007). En consecuencia, numerosos estudios han demostrado el papel que juega la inteligencia emocional en las reacciones emocionales subjetivas de un individuo cuando se enfrenta a acontecimientos estresantes y cómo estas habilidades emocionales pueden influir en el bienestar subjetivo (Salovey, Mayer, Caruso y Yoo, 2009).

Fernández-Berrocal y Extremera (2010) afirman que el concepto de inteligencia emocional se refiere a las diferencias individuales en la percepción, el procesamiento, la regulación y la utilización de la información emocional. Merece la pena analizar estas competencias que han demostrado tener un impacto significativo sobre la salud física y mental (Extremera y Fernández-Berrocal, 2006); el desempeño laboral y las relaciones sociales (Nelis, Quoidbach, Mikolajczak y Hansenne, 2009).

\subsection{Competencias de la inteligencia emocional}

La importancia de estos resultados ha generado diferentes modelos de la inteligencia emocional (Cherniss y Goleman, 2001). Según la Enciclopedia de Psicología Aplicada (Spielberger, 2004) se establecen tres modelos conceptuales: el modelo de Salovey y Mayer (1990), el modelo de Goleman $(1996,1999)$ y el modelo de Bar-On (2006).

En la teoría formulada por Salovey y Mayer (1990), la inteligencia emocional se enmarca dentro de un modelo de habilidad. Por otro lado, Goleman (1996, 1999) entiende la inteligencia emocional como un conjunto de competencias en las que se recogen el autocontrol, la perseverancia, la automotivación y la capacidad para retrasar la gratificación. Mientras que Bar-On (1988) ha colocado a la inteligencia emocional en el contexto de la teoría de la personalidad, específicamente en un modelo de bienestar.

Mayer y Salovey (1990) entienden la inteligencia emocional como la capacidad de controlar los propios sentimientos y emociones y los de los demás, haciendo uso de 
las mismas para guiar el pensamiento y la acción. El modelo presentado enfatiza en los aspectos cognitivos relacionados con el reconocimiento de las emociones, ya que la inteligencia emocional es concebida como la habilidad de percibir, comprender, utilizar y manejar las emociones para facilitar el pensamiento (Mayer, Salovey y Caruso, 2004). El modelo teórico se compone de habilidades: percepción de emociones; facilitación emocional; comprensión emocional y manejo de emociones.

Por su parte, Goleman (1996) identifica cinco competencias dentro de la inteligencia emocional: conocer las emociones, reconocerlas e identificarlas en uno mismo, gestionarlas, dominando las negativas y sustituyéndolas por positivas, la automotivación, como la capacidad de ilusionarse con cualquier situación salvo que sea objetivamente negativa en cuyo caso mejor alejar los pensamientos de la misma y reconocer emociones en otros y la empatía, como la capacidad de ponerse en la situación emocional de otro.

Bar-On $(2002,2006)$ define la inteligencia emocional como el conjunto de competencias emocionales, personales e interpersonales que determinan nuestra habilidad para afrontar los desafíos diarios. Esta inteligencia es un factor clave para tener éxito en la vida e influye directamente en el bienestar de las personas. Las personas emocionalmente inteligentes son capaces de reconocer y expresar sus emociones, de actualizar sus capacidades potenciales, de comprender cómo se sienten otras personas, de mantener relaciones interpersonales satisfactorias. Son optimistas, flexibles, realistas, afrontan el estrés sin perder el control y, en definitiva, llevan una vida saludable y feliz. El modelo de Bar-On está formado por cinco componentes clave: intrapersonal, interpersonal, adaptabilidad, gestión del estrés y estado de ánimo.

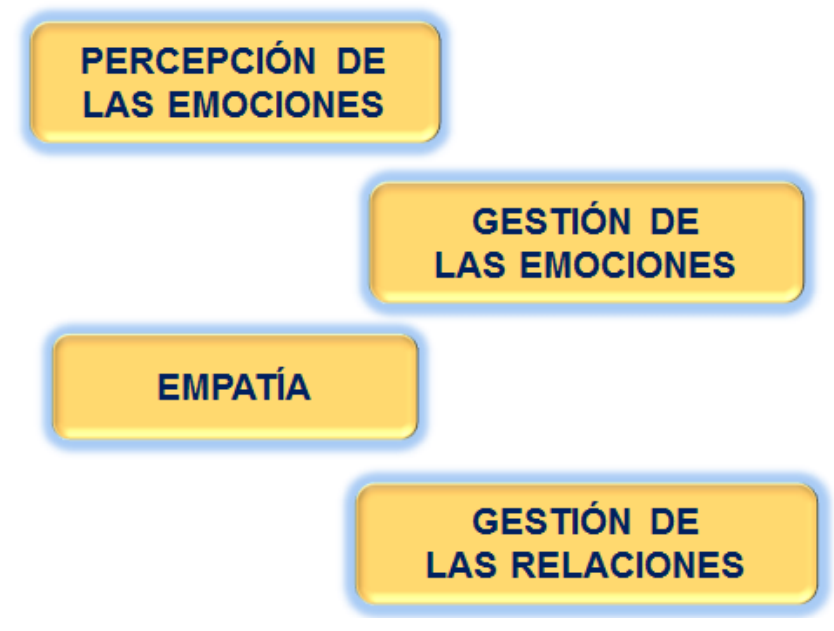

Figura 2. Competencias de la inteligencia emocional

En la figura 2, se presentan las competencias claves de la inteligencia emocional que se recogen en los tres modelos descritos que son la percepción de las propias emociones, la gestión útil de las emociones positivas y negativas, la empatía como la capacidad de percibir las emociones de los demás y la gestión inteligente y satisfactoria de las relaciones, aunque los términos utilizados para referirse a ellos sean diferentes (Cherniss y Goleman, 2001).

Sintetizando las ideas de los tres modelos, la inteligencia emocional es la capacidad del individuo para reconocer y regular las emociones en uno mismo y los 
demás en la búsqueda del bienestar personal y social. En la figura 3, se observa cómo el desarrollo de cada una de sus competencias (percepción, de las emociones, gestión, empatía y gestión de las relaciones) desarrollará una mayor inteligencia emocional. Entre las consecuencias positivas de esta mejora, diferentes estudios que se han ido citando muestran un mayor control de los pensamientos, toma de decisiones más útiles, hábitos saludables, autoestima y más éxito personal, social, académico y profesional. Todas estas consecuencias tienen una relación directa con el concepto de salud de la Organización Mundial de la Salud que se resume en un mayor bienestar físico, mental y social.

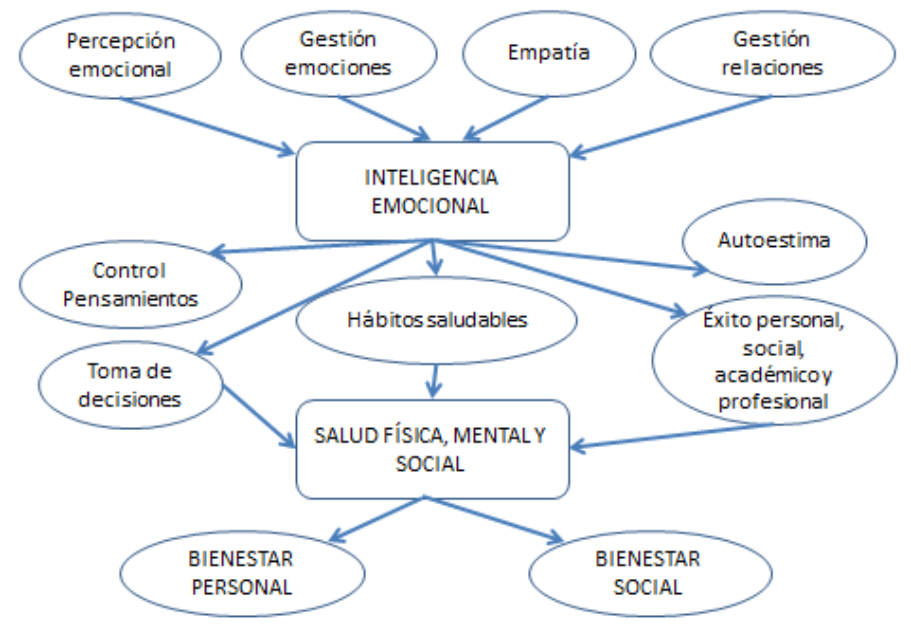

Figura 3. Desarrollo y consecuencias saludables de la inteligencia emocional.

Entre los hábitos saludables que se relacionan con la inteligencia emocional, nos detenemos en analizar su relación con la actividad físico-deportiva.

\section{ACTIVIDAD FÍ́SICO-DEPORTIVA E INTELIGENCIA EMOCIONAL}

Como se ha comentado, las emociones tienen una respuesta fisiológica en el organismo, ya que se instalan en la mente y en el cuerpo (Davidson, 2012). Por esta razón, a veces nos entran agujetas de reírnos, otras veces sudamos a causa del miedo o de ansiedad o aumenta nuestra frecuencia cardiaca cuando tenemos estrés o nos enfadamos. Punset Bannel (2012) comenta la comunicación no verbal como ejemplo de expresar emociones a través del movimiento. Conocer más profundamente la relación entre las emociones y la actividad física es el objetivo de este apartado.

\subsection{Qué ocurre cuando practicamos Actividad Físico-Deportiva}

Los beneficios de la actividad física han sido ampliamente estudiados (Marquez, Rodríguez y De Abajo, 2006). En los diferentes capítulos de este libro aparecen numerosas evidencias. Está en el primer nivel dentro de las recomendaciones que cualquier experto daría como medicina preventiva. Su relación con los beneficios fisiológicos (sistema cardio-vascular, colesterol, etc.) ha sido la más estudiada en las últimas décadas. Sin embargo, su relación con las emociones ha sido menos estudiada 
y supone una línea de investigación reciente. Ratey (2008) demuestra que cada día hay más evidencias de cómo el ejercicio contribuye a regular el cerebro emocional. Davidson (2012) llega a afirmar que las emociones son la forma más corporal del cerebro debido a cómo éstas afectan a la fisiología y cómo el movimiento afecta al modo en que la mente procesa la información emocional. Para empezar, como comenta Bilbao (2013), las neuronas son devoradoras de oxígeno, por lo que el cerebro necesita un corazón fuerte y unas arterias en perfecto estado para funcionar a pleno rendimiento. Por esta razón, este autor confirma que el ejercicio aeróbico moderado realizado regularmente es un factor de primer nivel para evitar el deterioro del cerebro, la muerte neuronal y otras alteraciones asociadas al envejecimiento. Davidson (2012) recuerda que el cerebro es el encargado de todo cuanto tiene que ver con la conducta humana, incluyendo la gestión de las emociones. Por tanto, la actividad física ayuda a mantener el cerebro sano y a que sus funciones, incluyendo la gestión emocional, se realicen adecuadamente.

La relación del ejercicio físico con el cerebro va más allá del aporte de oxígeno. El neurocientífico Fernández-Pinilla (Redes, 2010) explica que la actividad física favorece la secreción de la proteína BDNF que estimula el crecimiento neuronal. Esta proteína ayuda a crear nuevas sinapsis y nuevas redes neuronales que influyen directamente en el desarrollo intelectual en el más amplio sentido del término. Probablemente, el aumento de este neurotransmisor explica el estudio citado por Punset Bannel (2012) en el que solo por caminar 20 minutos diarios, una muestra de niños aumentó su rendimiento académico un $15 \%$ Es decir, la proteína BDNF ayuda al desarrollo de nuevos aprendizajes, así como a aprender de los errores o a adaptarse al cambiante mundo que nos rodea. Estas últimas ideas tienen una relación directa con la inteligencia emocional como hemos visto en el apartado anterior.

La actividad física supone un apoyo importante tanto en el fortalecimiento de emociones positivas como en la evitación de emociones negativas (Bilbao, 2013). Al realizar cualquier tipo de actividad física, el organismo segrega hormonas y neurotransmisores (endorfina, serotonina) que nos hacen sentir bien. Aumenta el nivel de energía de nuestro organismo. Esta sensación suele tenerse durante la realización de la actividad ya que el efecto de las endorfinas es rápido y potente. En ocasiones, ante un ejercicio duro durante su realización podemos experimentar cierto malestar 0 cansancio por el esfuerzo. Sin embargo, todos los que hemos hecho actividad física intensa, al finalizar y tras una reconfortante ducha, la sensación de bienestar y de relajación es definitivamente recomendable. Estas hormonas que se producen al practicar actividad física (también al comer, al reír, al estar con amigos, etc.) son llamadas hormonas de la felicidad (Bilbao, 2013). La práctica de actividad físicodeportiva nos hace sentirnos mejor, estar más felices, además de aportar vitalidad y buen ánimo. En esta línea, Molina-García, Castillo, Queralt (2011) encontraron que los mayores niveles de actividad física se asociaban con altos índices de bienestar psicológico. En concreto, los jóvenes con altos niveles de actividad presentaban mayor vitalidad subjetiva. Por su parte, Haugen, Säfvenbomb, y Ommundsena (2011) concluyeron que el aumento de los niveles de actividad física puede ser beneficioso dada la mejora de percepción de la autoestima física. En este sentido, diferentes estudios muestran que aparte de la modalidad de ejercicio practicado, éste contribuye de forma notable en la mejora del bienestar psicológico y/o emocional (Janisse, Nedd, Escamilla, y Nies, 2004; Wang et al., 2010; Zanuso, Sieverdes, Smith, Carraro, y Bergamin, 2012), sin que la duración ni intensidad sean factores que medien (Szabo, 2013).

La actividad física también influye en dos neurotransmisores que ayudan a regular los niveles de energía y de estrés que son la dopamina y la epinefrina. Ser una 
persona activa físicamente aumenta los niveles de dopamina previniendo los efectos adversos del agotamiento. La epinefrina nos ayuda a afrontar situaciones de estrés. Sin embargo, cuando el estrés es continuo, la presencia de este neurotransmisor agota al organismo y nos hace sentirnos cansados y desanimados. En este caso, el ejercicio físico moderado puede reducir los niveles de epinefrina y por tanto de estrés.

El neurólogo Álvaro Pascual-Leone (Redes 2013) comenta que el ejercicio físico activa zonas cerebrales que permiten tener mayor control inhibidor, mayor capacidad de resistir tentaciones, mayor capacidad de tomar decisiones a su tiempo en lugar de aceleradamente. Es decir, realizar unos 20 minutos de actividad física aeróbica diariamente modifica nuestro cerebro en una forma beneficiosa.

La relación de la actividad física con la gestión de las emociones negativas es también muy útil. La práctica de un deporte o un baile bloquea los pensamientos negativos o preocupaciones que se pudieran tener, ya que exige al cerebro una ocupación total en la actividad con el organismo generando endorfinas. El ejercicio físico también contribuye a dominar el enfado (Goleman, 1996). Por una parte, hace que disminuya la elevada excitación provocada por la ira, damos tiempo a que el cerebro se tranquilice, se ocupe de otra cosa y relativice. Starks, Starks, Kingsley, Purpura y Jager (2008) demuestran que el ejercicio físico moderado por debajo de 40-45 minutos disminuye los niveles de cortisol. En definitiva, supone una excelente herramienta para disminuir el estrés.

Si llevar una vida activa tiene unas recomendables consecuencias positivas, el sedentarismo, como afirma Ratey (2008), supone un trastorno a nuestra naturaleza cuyas consecuencias sobre la salud son muy importantes, incluida la salud emocional. De forma inversa, la falta de ejercicio influye negativamente sobre nuestra energía, nuestro estado de ánimo, el sistema inmunológico y por tanto en prevenir, curar o retrasar enfermedades. La razón es muy sencilla tal y como la expone Punset Bannel (2012): nuestros genes se desarrollaron hace más de medio millón de años cuando nos desplazábamos una media de 15 kilómetros diarios y hacíamos ejercicio constantemente. Nuestros genes son los mismos y necesitan movimiento porque forma parte de nuestro cerebro. De hecho, como afirma Wolper (2011), la verdadera razón de tener cerebro es el movimiento. El estilo de vida actual ha reducido de forma muy peligrosa para la salud, los niveles adecuados de actividad física que necesita nuestro cerebro para un funcionamiento óptimo.

En definitiva, la relación de la actividad física con el estado emocional es evidente. En el gráfico 4, se resumen los beneficios. La mayor oxigenación del cerebro que produce el ejercicio físico lo protege del envejecimiento, reduciendo el riesgo de enfermedades neurodegenerativas y aportando más recursos para retrasar sus efectos. Por otra parte, las hormonas del bienestar que se segregan durante y después de practicar actividad físico-deportiva hacen sentirnos bien, nos aporta vitalidad y energía, reduce el estrés al ayudar a eliminar hormonas como el cortisol, aumenta nuestra autoestima, la percepción satisfactoria de nuestra imagen corporal y se duerme mejor. Asimismo, la mayor producción del neurotransmisor BDFN aumenta nuestra capacidad de aprender y por tanto, el rendimiento académico, estimula zonas de nuestro cerebro que se encargan del autocontrol y de la toma de decisiones calmada y, en definitiva, nos dota de más competencias para adaptarnos a diferentes situaciones. 


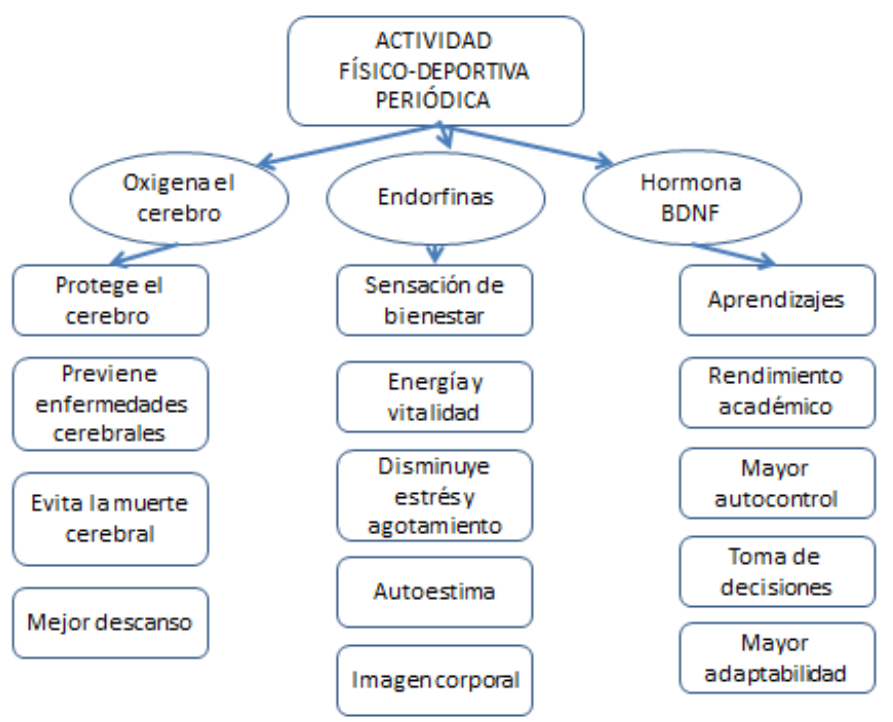

Figura 4. Beneficios cerebrales y emocionales de la actividad física

\subsection{Inteligencia emocional y actividad física}

Ante estas evidencias, es lógico que recientemente aparezca una línea de investigación en la psicología del deporte que correlacione estas dos variables, aunque el número de estudios es reducido. Fernández-Ozcorta (2013) demostró que los jóvenes universitarios activos presentaron mayores niveles de inteligencia emocional. Parece que el efecto derivado de la realización de actividad física, en niveles adecuados, tiene un efecto positivo sobre las variables de claridad emocional, reparación emocional, así como sobre la autoestima y la satisfacción con la vida. Es decir, la actividad física de forma general, sirve de soporte para tener mayor grado de bienestar psicológico de los estudiantes. Esta relación entre la actividad física y la inteligencia emocional ha sido mostrada en otros estudios como los de Li, Lu, y Wang (2009); Saklofske, Austin, Rohr, y Andrews (2007) o Tsaousis y Nikolaou (2005). Brown y Schutte (2006) encontraron una relación inversamente proporcional entre los niveles inteligencia emocional y la fatiga subjetiva o cansancio en estudiantes universitarios. De hecho, Núñez, León, González y Martín-Albo (2011) plantean la duda de que variable (actividad física e inteligencia emocional) tiene más poder predictor sobre la otra.

Un desarrollo de la inteligencia emocional percibida provee al individuo de la capacidad de gestionar las emociones subjetivas cuando se en enfrenta a situaciones estresantes (Salovey et al., 2009). Por lo que parece que un desarrollo correcto de la inteligencia emocional permitirá gestionar más eficazmente las situaciones negativas que se produzcan en el contexto deportivo, además de contar con mayores estrategias de afrontamiento más adaptativas y superior la capacidad de resolución de problemas sociales (Saklofske et al., 2007). La mejora de la eficacia deportiva a través de un desarrollo de la inteligencia emocional ha sido también estudiado (Kouli, Bebetsos, Kamperis y Papaioannou, 2010; Puig, 2012). El papel de los psicólogos deportivos en diferentes deportes de alta competición es cada vez más importante y gran parte del trabajo que realizan se centra en la mejora de competencias emocionales.

\subsection{Qué emociones se experimentan en función del tipo de práctica}

Estos beneficios que se han estado citando, se consiguen con cualquier tipo de actividad física, realizada a una adecuada intensidad y duración. Otra línea de 
investigación se plantea qué tipo de emociones se despiertan en función de la actividad desarrollada. Se parte de la base de que el juego motor, el deporte, el baile, etc. son actividades emocionantes. En algunos casos como el deporte, se muestran emociones muy visibles y muy intensas como la alegría, la ira, el miedo o cuando en el baile que puede aparecer la diversión, la vergüenza o la nostalgia. Conocer el tipo de emociones y la intensidad que generan los juegos y actividades motrices ha sido una línea de trabajo con interesantes repercusiones educativas y saludables (Alonso, Gea, y Yuste, 2013; Lavega, Aráujo, y Jaqueira, 2013; Lavega, Filella, Agulló, Soldevilla, y March, 2011). Para ello, clasifican las actividades en cuatro: individuales sin oposición, individuales con oposición, cooperativas sin oposición y cooperativas con oposición y tras practicar diferentes juegos de cada tipo, los participantes muestran su percepción sobre las emociones experimentadas y su intensidad.

En general, sintetizando los resultados de los estudios citados, las actividades físicas y deportivas generan una intensidad más alta de emociones positivas que de negativas y ambiguas, debido a los efectos que los neurotransmisores producen en el cerebro. No obstante, se encuentran diferencias en función del tipo de acción motriz experimentada. Así, las actividades individuales sin oposición suelen presentar niveles más bajos de emociones positivas y negativas que cuando hay oposición. Por su parte, las actividades de colaboración desarrollan emociones positivas más intensas que las individuales. Las de cooperación-oposición suelen ser las más intensas, aunque también aparecen más emociones negativas que en las individuales. Según Bisquerra (2000), la interacción con las personas y el ambiente es lo que nos genera las emociones y las personas son los elementos esenciales, quizás podría ser ese efecto socializador el que hace que sentimos con más intensidad las emociones.

La competición en forma de oposición es un factor que intensifica las emociones tanto positivas como negativas, aunque siempre las positivas se valoran por encima (Alonso, Gea, y Yuste, 2013). Las actividades de cooperación sin oposición son las que suelen presentar los valores menos intensos en emociones negativas.

Los alumnos que tienen antecedentes deportivos sienten con más intensidad las emociones positivas y ambiguas. Asimismo, los practicantes de deportes sociomotrices valoran más intensamente las emociones comparados con los que han practicado deportes individuales.

En cualquier caso, los estudios demuestran que cada uno de los tipos de acciones motrices producen emociones diferentes tanto en cada persona como, globalmente, por lo que producen las variables de relación (individual o colectivo) o de competición (con oposición o sin oposición). Los resultados son interesantes a la hora de selección el tipo de tarea en función de las emociones que se persigan.

\subsection{Una relación muy saludable}

Habiendo analizado los beneficios que aportan la actividad física y la gestión inteligente de las emociones a la salud, es de sentido común que aquellas personas que aumenten los niveles de ambos parámetros, conseguirán una mayor calidad de vida. Si unos niveles altos de inteligencia emocional correlacionan positivamente con niveles altos de bienestar, el nivel de actividad física puede modificar aún más la percepción del bienestar experimentado (Núñez et al., 2011). Es decir, los niveles altos y adecuados de ejercicio físico, junto con niveles altos de inteligencia emocional, aumentan la autoestima, la satisfacción con la vida y la gestión de las emociones. 


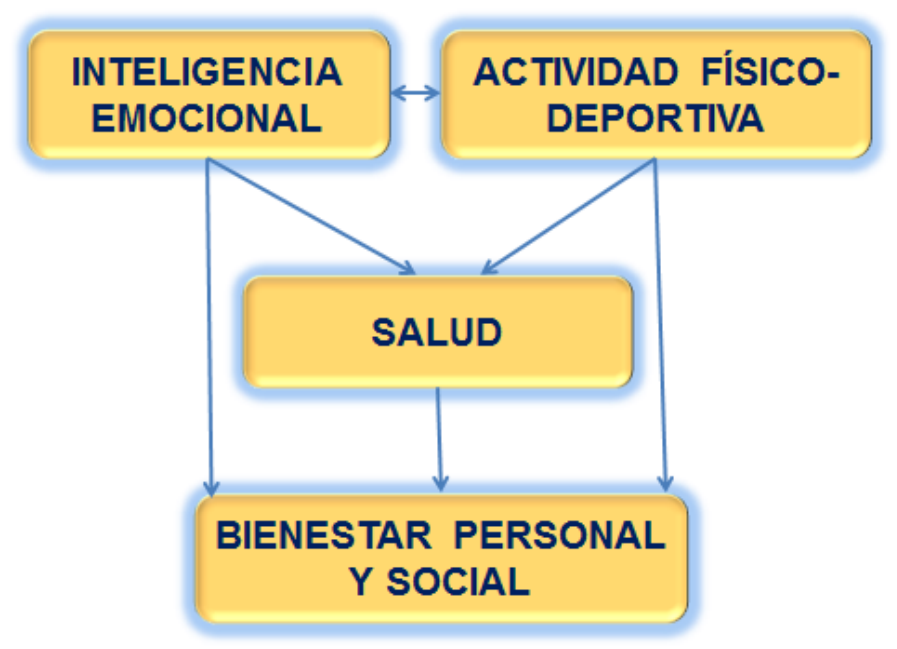

Figura 5. Relación de la actividad física y la inteligencia emocional con el bienestar

Resulta útil comprobar la relación de la inteligencia emocional y el bienestar con la motivación en contextos de práctica físico-deportiva. Núñez et al. (2011) y Fernández (2013) incluyeron la inteligencia emocional percibida junto a otras variables motivacionales para explicar el bienestar psicológico en el marco de la teoría de metas de logro y la teoría de la autodeterminacón, dentro de diferentes contextos deportivos. Estos autores, destacan que la inteligencia emocional percibida jugaba un papel importante dentro de un proceso de motivación deportiva. Estos estudios muestran cómo la inteligencia emocional percibida tiene un valor predictivo sobre la satisfacción de las necesidades psicológicas básicas (autonomía, competencia y relaciones). Está ampliamente estudiada la influencia que estas tres necesidades tienen en una mayor motivación autodeterminada hacia a la actividad físico-deportiva (Deci y Ryan, 2000). Ya se ha comentado la capacidad de la inteligencia emocional percibida de predecir de forma positiva, la autoestima y la satisfacción con la vida (Rey et al., 2011). El hecho de que la satisfacción de las necesidades psicológicas básicas sea también mediador entre la inteligencia emocional y el bienestar psicológico (Meyer, Enströma, Harstveita, Bowlesb, y Beeversc, 2007; Núñez et al., 2011; o Fernández-Ozcorta, 2013), aporta estrategias para aumentar la motivación intrínseca y la adherencia hacia la práctica deportiva (Almagro, Sáenz-López, y Moreno, 2010; Moreno, Conte, Borges y GonzálezCutre, 2008), con el fin de conseguir una población cada vez más activa, cada vez más saludable y cada vez más feliz. Torregrosa, Belando y Moreno-Murcia (2013) concluyen que el estilo controlador de los docentes predijo negativamente la satisfacción con la vida, mientras que el apoyo a la autonomía y las necesidades psicológicas básicas predijeron positivamente. A modo de ejemplo, cuanta más autonomía, es decir más libertad para tomar decisiones durante la práctica de actividad física, cuanta más percepción de competencia a través de actividades que hagan sentirse eficaces 0 siendo recompensados por las cosas bien hechas y cuanta más y mejores relaciones se establezcan durante la práctica deportiva, mayor será la motivación y las probabilidades de seguir practicando. 


\section{CONCLUSIONES Y RECOMENDACIONES}

\subsection{Objetivos planteados}

El primer objetivo fue analizar la relación entre la inteligencia emocional y la actividad físico-deportiva.

- El ejercicio físico produce hormonas que nos hacen sentir bien.

- La práctica periódica de actividad física aumenta la autoestima y la satisfacción con nuestra imagen corporal.

- Las personas activas físicamente presentan niveles más elevados de algunas competencias de la inteligencia emocional como el autocontrol, la gestión de emociones o las relaciones sociales.

- La práctica de juegos motrices es emocionante. Prevalecen las emociones positivas sobre las negativas con más intensidad cuando hay oposición/competición.

- La inteligencia emocional permite una práctica deportiva más eficiente.

El segundo objetivo pretendía valorar la importancia de la actividad física y la inteligencia emocional en la salud preventiva y su influencia en el bienestar.

- La actividad física permite una mayor oxigenación del cerebro lo que permite una mayor protección del mismo hacia enfermedades degenerativas.

- El ejercicio físico disminuye el estrés y el agotamiento ya que elimina toxinas y dota de mayor vitalidad al organismo.

- La práctica de actividad físico-deportiva facilita la producción una hormona que ayuda a tomar decisiones más inteligentes, facilita aprendizajes cognitivos y aumenta la capacidad de adaptarnos a situaciones diferentes.

- La inteligencia emocional ayuda a controlar los pensamientos y a tomar decisiones más inteligentes.

- Mayores niveles de inteligencia emocional garantizan mejores hábitos saludables como el menor consumo de tabaco o alcohol y mayor práctica de ejercicio físico.

- La inteligencia emocional se relaciona con un mayor éxito académico, profesional y de relaciones sociales.

Mayores niveles de inteligencia emocional correlacionan positivamente con mayores niveles de bienestar personal y social. El nivel de actividad física influye directamente en el mayor bienestar y combinado con los niveles de inteligencia emocional parece que lo mejora todavía más.

El tercer objetivo era realizar propuestas para desarrollar una actividad física emocionante y saludable en busca del bienestar personal y social. En el apartado siguiente, se exponen una serie de recomendaciones basadas en las evidencias que se han ido mostrando a lo largo del capítulo.

\subsection{Recomendaciones para una actividad física emocionante y saludable}

\section{Recomendaciones a nivel personal}

Los hábitos de vida son, como afirma Bilbao (2013), pautas de comportamiento que se han ido aprendiendo durante periodos prolongados. Generar un estilo de vida más saludable física y emocionalmente para conseguir mayor bienestar, requiere generar nuevos hábitos y aquí está la clave del éxito. Los seres humanos funcionamos a través rutinas que algunos autores denominan como "zona de confort" (Punset Bannel, 
2012). Salir de la zona de confort y generar nuevos hábitos requiere intención, práctica y éxito. Vamos a enumerar una serie de recomendaciones que pueden ayudar a mejorar nuestra inteligencia emocional y a aumentar nuestro nivel de práctica de actividad física.

- Ten claro lo que quieres.

Plantearse objetivos, saber qué quieres, es esencial.

- Imagina mentalmente lo que quieres. En el caso de la inteligencia emocional, ser más tranquilo, sonreír más, tener mejores relaciones, etc. En el caso de la actividad física a qué aspiras, a correr media hora diaria, a hacer deporte con los amigos, a bailar, etc. Tenlo claro.

Escribe en un papel estos objetivos, te ayudará.

- El objetivo final mínimo es hacer al menos 20 minutos diarios de actividad física de cierta intensidad aeróbica.

- Hazlo público de forma positiva.

Comentar tus intenciones te ayudará a conseguir los cambios. Aquellas personas que hacen públicos sus compromisos tienes más probabilidades de éxito.

Comenta con la familia y amigos tus objetivos y tus logros.

Escríbelos en un papel y ponlos en la nevera o tu lugar de trabajo.

Hazlo siempre de forma positiva. Plantea el reto y se consiga o no, piensa y comenta lo hecho en lugar de lo que ha faltado. Comenta los beneficios de la actividad física y la inteligencia emocional. Es mejor pensar en los aspectos positivos que en los negativos.

- El siguiente reto que se fácil.

Márcate objetivos realizables en función de tus capacidades. Andar 15 minutos es un reto para un sedentario. Los beneficios merecen la pena. Márcate el siguiente reto que puede ser aumentar un poco la intensidad o la frecuencia. Con esta forma de establecer objetivos estás trabajando la inteligencia emocional.

Establece una rutina sencilla y realízala durante 21 días para que se haga hábito. Andar, subir las escaleras o evitar enfadarme. Busca un momento del día para dedicarlo a este objetivo.

Busca una afición que te apasione y practícala sistemáticamente.

- Aprovecha cualquier oportunidad para hacer ejercicio. Subir escaleras en lugar de ascensor, ir andando en lugar de en coche, coger la bicicleta con más frecuencia, sacar a pasear al perro...

- Baila un rato todos los días. Ponte música o canta y baila unos minutos.

- Si tienes un momento malo, te surge una emoción negativa por cualquier problema, lo más recomendable es hacer unos minutos de actividad física. Se eliminarán las toxinas y podemos permitir que las endorfinas nos hagan sentir mejor.

- Reconoce tus emociones cuando practicas deporte; gestiona con inteligencia las negativas y potencia las positivas (evita tener pensamientos negativos).

Elige actividades que despierten emociones positivas.

Estimula todos los sentidos para desarrollar zonas del cerebro diferentes. Por ejemplo, huele las bebidas o los alimentos, toca objetos con diferentes partes 
del cuerpo, haz cosas cotidianas con los ojos cerrados como vestirse o hacer el amor.

- Consigue siempre éxito

La percepción de competencia es clave para la motivación, por tanto,

- Practica actividades adecuadas a tu nivel para que experimentes éxito.

- Céntrate y prémiate los avances, lo que consigues, en lugar de lamentarte por lo que no se ha realizado o cuando ha salido mal.

- Si practicas deporte, fomentar el altruismo y la compasión nos hace sentirnos mejor y nos hace sentirnos competentes.

- También ayuda la auto-responsabilidad. Evitar echar la culpa de lo que me pasa a otros o a las circunstancias. Es más saludable que, ante cualquier conflicto, practiquemos el centrarnos en nuestra responsabilidad.

- Rodéate de gente positiva y activa

Las relaciones satisfactorias son otra necesidad psicológica básica para aumentar la motivación intrínseca.

- Busca amigos que les guste practicar deporte o salir a pasear o hacer excursiones.

- Establece un horario para practicar deporte con amigos como jugar al padel o al fútbol.

- Fomenta actividades familiares activas como salir en bici, hacer excursiones al campo, apuntarse a un centro deportivo, etc.

- Apaga la televisión y juega con tus hijos o familiares.

- En todos los casos, fomenta el buen humor y conversaciones positivas y optimistas. Las emociones son contagiosas, por tanto, si tú estás positivo y sonriente, los demás tenderán a estarlo.

- Autonomía

A pesar de la importancia de las amistades, la práctica de actividad física depende sólo de uno.

Evita depender de la gente.

- Si haces actividades en grupo, que sea suficientemente amplio para que la actividad siempre se haga aunque falle alguno.

- Busca actividades físicas en las que tengas que tomar decisiones. Por ejemplo, cualquier deporte. En caso de prácticas individuales, ten autonomía y decisión para controlar la frecuencia, la intensidad, etc.

- Disfruta

La pasión y el disfrute es clave para el bienestar.

Busca actividades físicas placenteras, que te gusten y que se te den bien. 
- Disfruta de los preparativos. Piensa y comenta cuándo vas a hacer actividad física y hazlo con ilusión. Convéncete que haces actividad física porque quieres, porque disfrutas mucho más que por sus beneficios.

Disfruta del proceso. Durante la práctica, sonríe, céntrate en tus movimientos, en tu entorno, siente tu corazón, tus músculos moviéndose...

Aprende a relajarte.

Después de hacer actividad física, relájate, siente cómo las endorfinas hacen su trabajo y te hacer sentir bien.

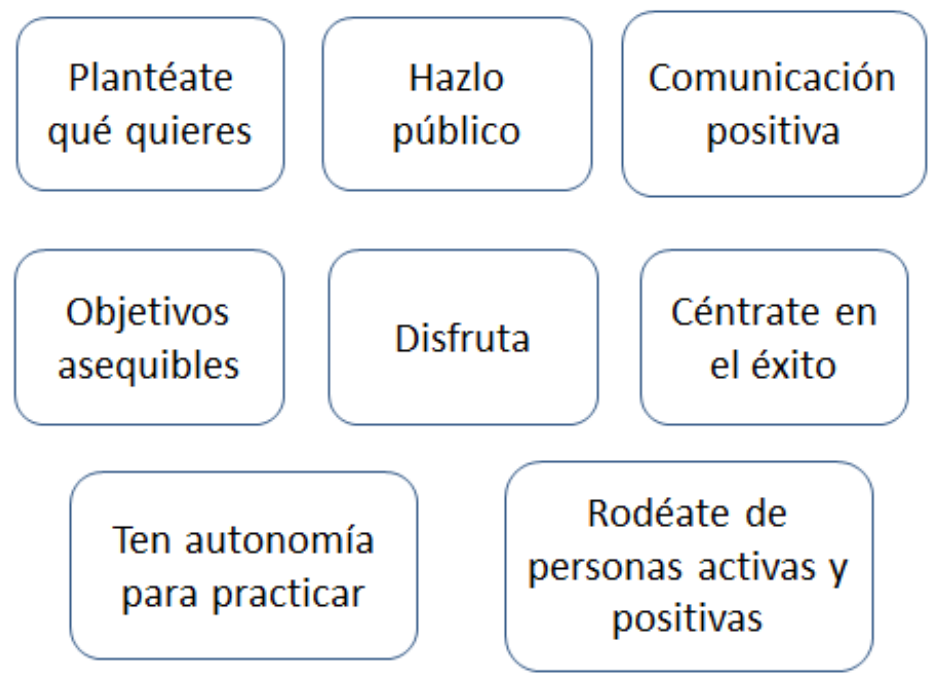

Figura 6. Recomendaciones para una actividad física emocionante y saludable

\section{Recomendaciones a nivel institucional}

Las instituciones responsables del bienestar (educación y sanidad) tienen un reto en la búsqueda del bienestar. Siguiendo los siete pilares de las recomendaciones a nivel personal, los centros educativos, patronatos o instalaciones deportivas necesitan: tener los objetivos muy claros, lanzar mensajes positivos, facilitar la práctica a toda la población, disponer de docentes y monitores que fomenten la autonomía y la percepción de competencia de los participantes, crear climas de trabajo positivos, fomentar la autonomía y, sobre todo, hacer disfrutar de la práctica de actividad físico-deportiva.

\section{REFERENCIAS BIBLIOGRÁFICAS}

Almagro, B. J., Sáenz-López, P., y Moreno, J. A. (2010). Prediction of sport adherence through the influence of autonomy-supportive coaching among Spanish adolescent athletes. Journal of Sports Science and Medicine, 9, 8-14.

Alonso, J. I., Gea, G., \& Yuste, J.L. (2013). Formación emocional y juego en futuros docentes de educación física. Revista Electrónica Interuniversitaria de Formación del Profesorado, 16(1), 97-108. http://www.aufop.com/aufop/uploaded_files/articulos/1375389683.pdf

Austin, E. J., Saklofskeb, D. H. y Eganc, V. (2005). Personality, well-being and health correlates of trait emotional intelligence. Personality and Individual Differences, 38, 547-558. 
Bar-On, R. (1988). The development of an operational concept of psychological well-being [Documento en linea]. Unpublished doctoral dissertation, Rhodes University: Sudáfrica. Disponible en: http://eprints.ru.ac.za/1994/1/BAR-ON-PhD-TR89-44.pdf

Bar-On, R. (2002). Baron Emotional Quotient Inventory: Short (BarOn EQ-i:S) technical manual. Toronto, ON: Multi-Health Systems Inc.

Bar-On, R. (2006). The Bar-On model of emotional-social intelligence (ESI). En P. FernándezBerrocal y N. Extremera (Eds.), Special Issue on Emotional Intelligence. Psicothema, $18,13-25$.

Bilbao, A. (2013). Cuida tu cerebro... y mejora tu vida. Barcelona: Plataforma.

Bisquerra, R. (2000). Educación Emocional y bienestar. Barcelona: Praxis

Brown, R. F., y Schutte, N. S. (2006). Direct and indirect relationships between emotional intelligence and subjective fatigue in university students. Journal of Psychosomatic Research, 60, 585-593.

Cacioppo, J. T. y Patrick, W (2008). Human Nature and the Need for Social Connection. New York: Norton and Company.

Cherniss, C., y Goleman, D. (2001). The emotionally intelligent workplace. San Francisco, CA: John Wiley \& Sons.

Ciarrochi, J. V., Chan, A. Y. C., y Caputi, P. (2000). A critical evaluation of the emotional intelligence construct. Personality and Individual Differences, 28, 539-561.

Colegio Oficial de Psicólogos de Madrid (2014). Proyecto "emociones y salud". http://www.copmadrid.org/webcopm/resource.do?recurso=20111223124830109000

Damasio, A. R. (2001). El error de Descartes. Barcelona: Crítica.

Davidson, R. J. (2012). El perfil emocional de tu cerebro. Claves para modificar nuestras actitudes y reacciones. Barcelona: Destino.

Deci, E. L., y Ryan, R. M. (2000). The "what" and "why" of goal pursuits: Human needs and the self-determination of behaviour. Psychological Inquiry, 11, 227-268.

Diener, E. (1994). Assessing subjective well-being: Progress and opportunities. Social Indicators Research, 31, 103-157.

Extremera, N. y Fernández-Berrocal, P. (2004). El papel de la inteligencia emocional en el alumnado: evidencias empíricas. Revista Electrónica de Investigación Educativa, 6. Disponible en: http://redie.uabc.mx/vol6no2/contenido-extremera.html

Extremera, N., Ruiz-Aranda, D., Pineda, C., y Salguero, J. M. (2011). Emotional intelligence and its relation with hedonic and eudaimonic well-being: a prospective study. Personality and Individual Differences, 51, 11-16.

Extremera, N., y Fernández-Berrocal, P. (2006). Emotional Intelligence as Predictor of Mental, Social, and Physical Health in University Students. The Spanish Journal of Psychology, 9, 45-51.

Felten, D. L. y Cohen, N. (2006). Psychoneuroimmunology. New York. Academic press.

Fernández-Ozcorta, E. (2013). Factores motivacionales y su relación con la práctica de actividad física en el alumnado universitario de Huelva. Tesis doctoral inédita. Huelva.

Fernández-Berrocal, P. y Extremera, N. (2010). Más Aristóteles y Menos Prozac. Encuentros en Psicología Social, 5, 40-51.

Fernández-Berrocal, P., Ramos, N., y Extremera, N. (2001). Inteligencia emocional, supresión crónica de pensamientos y ajuste psicológico. Boletín de Psicología, 70, 79-95. 
Gagne, M., R.M. Ryan, y K. Bargmann (2003): «Autonomy support and need satisfaction in the motivation and well-being of gymnasts», Journal of Applied Sport Psychology, 15, 372390.

Gardner, H. (2001). Estructuras de la mente. Teoría de las inteligencias múltiples. Colombia: Fondo de Cultura Económica.

Goleman, D. (1996). Inteligencia emocional. Barcelona: Kairós.

Goleman, D. (1999). La práctica de Ia Inteligencia Emocional. Barcelona: Editorial Kairós.

Haugen, T., Säfvenbomb, R., y Ommundsena, Y. (2011). Physical activity and global self-worth: The role of physical self-esteem indices and gender. Mental Health and Physical Activity, 2, 49-56.

Heck, G. L., y Oudsten, B. L. (2008). Emotional intelligence: Relationship to stress, health, and well-being. En A. Vingerhoets, I. Nyklicek y J. Denollet (Eds.), Emotion regulation. Conceptual and clinical issues (pp. 97-121). Nueva York: Springer.

Instituto Nacional de Estadística (2013). Notas de prensa: Encuesta Nacional de Salud 20112012 [Documento en línea]. Madrid: Ministerio de sanidad, servicios sociales e igualdad. Disponible en: http://www.ine.es/prensa/np770.pdf

Janisse, H., Nedd, D., Escamilla, S., y Nies, M. (2004). Physical activity, social support, and family structure as determinants of mood among European-American and AfricanAmerican women. Women Health, 39, 101-116.

Kouli, O; Bebetsos, E.; Kamperis, I. y Papaioannou, A. (2010). The relationship between emotions and confidence among greek athletes from different competitive sports. Kinesiology, 42(2), 194-200.

Lavega, P.; Aráujo, P., y Jaqueira, A. R. (2013). Teaching motor and emotional competencies in university students. Cultura Ciencia y Deporte, 8(22), 5-15. http://ccd.ucam.edu/index.php/revista/issue/view/48

Lavega, P.; Filella, G.; Agulló, Ma․ J.; Soldevilla, A.; \& March, J. (2011). Understanding emotions through games: helping trainee teachers to make decitions. Electronic Journal of Research in Educational Psychology, 9(2), 617-640. http://www.investigacionpsicopedagogica.com/revista/articulos/24/english/Art_24_519.pdf

Li, G. S. F., Lu, F. J. H., y Wang, A. H. H. (2009). Exploring the relationships of physical activity, emotional intelligence and health in Taiwan college students. Journal of Exercise and Scientific Fitness, 1, 55-63.

Limonero, J. T, Tomás-Sábado, J. \& Fernández-Castro, J. (2006). Perceived emotional intelligence and its relation to tobacco and cannabis use among university students, Psicothema, 18, supl., 95-100.

Lopes, P. N., Salovey, P., y Straus, R. (2003). Emotional intelligence, personality and the perceived quality of social relationships. Personality and Individual Differences, 35, 641658.

Mayer, J. D., Salovey, P., y Caruso, D. R. (2004). Emotional intelligence: Theory, findings, and implications. Psychological Inquiry, 15, 197-215.

Mayer, J. D., y Salovey, P. (1997). What is emotional intelligence? En P. Salovey y D. Sluyter (Eds.), Emotional Development and Emotional Intelligence: Implications for Educators (pp. 3-31). Nueva York: Basic Books.

Marquez, S.; Rodríguez, J. y De Abajo, S. (2006). Sedentarismo y Salud: efectos beneficiosos de la actividad física. Apunts, 83, 12-24. 
McDowell, I. (2010). Measures of self-perceived well-being. Journal of psychosomatic research, $69,69-79$.

McGillivray, M. (2007). Human Well-being: Issues, Concepts and Measures. En M. McGillivray (Ed.), Human Well-Being: Concept and Measurement (pp. 1-22). Basingstoke, RU: Palgrave MacMillan.

Meyer, B. B., y Zizzi, S. (2007). Emotional intelligence in sport: conceptual, methodological, and applied issues. En A. M. Lane (Ed.), Mood and human performance: Conceptual, measurement, and applied issues (pp. 131-154). Nueva York: Nova Science.

Meyer, B., Enströma, M. K., Harstveita, M., Bowlesb D. P., y Beeversc, C. G. (2007). Happiness and despair on the catwalk: Need satisfaction, well-being, and personality adjustment among fashion models. The Journal of Positive Psychology, 2, 2-17.

Molina-García, J., Castillo, I., y Queralt, A. (2011). Leisure-time physical activity and psychological well-being in university students. Psychological Reports, 109, 453-460.

Moreno, J.A., Conte, L., Borges, F. and González-Cutre, D. (2008) Necesidades psicológicas básicas, motivación intrínseca y propensión a la experiencia autotélica en el ejercicio físico. Revista Mexicana de Psicología, 25(2), 305-312.

Nasser, V. (2010). The psychology of happiness and well-being. London: Nasser.

Nelis, D., Quoidbach, J., Mikolajczak, M., y Hansenne, M. (2009). Increasing emotional intelligence: (How) is it possible? Personality and Individual Differences, 47, 36-41.

Núñez, J. L., León, J., González, V., y Martín-Albo, J. (2011). Propuesta de un modelo explicativo del bienestar psicológico en el contexto deportivo. Revista de Psicología del Deporte, 20, 223-242.

Organización Mundial Salud (2010). Recomendaciones mundiales sobre Actividad física para la salud. Ginebra: OMS.

Piqueras, J. A.; Ramos, V.; Martínez, A.; Oblitas, L. F. (2009). Emociones negativas y su impacto en la salud mental y física. Suma Psicología, 16(2), 85-112.

Puig, N. (2012). Emociones en el deporte y sociología. Revista Internacional de Ciencias del Deporte, 28, 106-108.

Punset Bannel, E. (2012). Una mochila para el universo. 21 rutas para vivir con nuestras emociones. Barcelona: destino.

Punset Casals, E. (2010). Viaje a las emociones. Las claves que mueven el mundo: la felicidad, el amor y el poder de la mente. Barcelona: Destino.

Ratey, J. (2008). Spark: The Revolutionary New Science of Exercise and the Brain. New York: Little Brown and Company.

Redes (2010). Deporte para un cerebro más sano. Programa Redes, 72. http://www.redesparalaciencia.com/3918/redes/redes-72-deporte-para-un-cerebromas-sano

Redes (2013). Estimula tu cerebro para vivir más y mejor. Programa Redes, 163. http://www.rtve.es/alacarta/videos/redes/redes-estimula-tu-cerebro-para-vivir-masmejor/1921047/

Rey, L., Extremera, N., y Pena, M. (2011). Perceived emotional intelligence, self-esteem and life satisfaction in adolescents. Psychosocial Intervention, 20, 227-234.

Ryan, R. M., y Deci, E. L. (2001): «On happiness and human potentials: A review of research on hedonic and eudaimonic well-being», Annual Review of Psychology, 52, 141-166. 
Saklofske, D. H., Austin, E. J., Rohr, B. A., y Andrews, J. J. W. (2007). Personality, emotional intelligence and exercise. Journal of Health Psychology, 12, 937-948.

Salovey, P., Mayer, J. D., Caruso, D., y Yoo, S. H. (2009). The positive psychology of emotional intelligence. En S. J. Lopez y C. R. Snyder (Eds.), The handbook of positive psychology ( $2^{\circ}$ ed., pp. 237-248). Nueva York: Oxford University Press.

Salovey, P., Mayer, J. D., Goldman, S. L., Turvey, C., y Palfai, T. P. (1995). Emotional attention, clarity and repair: exploring emotional intelligence using the trait meta-mood scale. En J. W. Pennebaker (Ed.), Emotion, disclosure and health (pp. 125-154). Washington D. C.: American Psychological Association.

Salovey, P., Stroud, L. R., Woolery, A., y Epel, E. S. (2002). Perceived emotional intelligence, stress reactivity, and symptom reports: Further explorations using the Trait Meta-Mood Scale. Psychology and Health, 17, 611-627.

Salovey, P., y Mayer, J. D. (1990). Emotional intelligence. Imagination, Cognition, and Personality, 9, 185-211.

Sapolsky, R. (1994). ¿Por qué las cebras no tienen úlcera? Barcelona: Alianza.

Schutte, N. S., Malouff, J. M., Bobik, C., Coston, T. D., Greeson, C., Jedlicka, C., Rhodes, E., y Wendorf, G. (2001). Emotional intelligence and interpersonal relations. Journal of Social Psychology, 141, 523-536.

Sociedad española de medicina preventiva, salud pública e higiene (2014). Web oficial. http://www.sempsph.com/es/

Spielberger, C. D. (2004). Encyclopedia of applied psychology. Oxford, Boston: Elsevier Academic Press.

Starks, M. A.; Starks, S.; Kingsley, M.; Purpura, M. y Jager, R. (2008). The effects of phosphatidylserine on endocrine response to moderate intensity exercise. Journal of the International Society of Sports Nutrition, 5: 11.

Szabo, A. (2013). Acute psychological benefits of exercise: Reconsideration of the placebo effect [Abstract]. Journal Mental Health, 0, 1-7.

Szabo, A., y Abrahám, J. (2012). The psychological benefits of recreational running: A field study. Psychology Health \& Medicine, 18, 251-261.

Torregrosa, M.; Belando, N. y Moreno-Murcia, J. A. (2013). Predicción de la satisfacción con la vida en practicantes de ejercicio físico saludable. Cuadernos de Psicología del Deporte, 14(1), 117-122.

Tsaousis, I., y Nikolaou, I. (2005). Exploring the Relationship between Emotional Intelligence and Physical and Psychological Health. Stress and Health, 21, 77-86.

Wang, Ch., Bannuru, R., Ramel, J., Kupelnick, B. Scott T., y Schmid, C. H. (2010). Tai Chi on psychological well-being: systematic review and meta-analysis. BMC: Complementary and Alternative Medicine, 10, 1-16

Wolper, D. (2011). The real reason for brains. TED. http://www.ted.com/talks/daniel_wolpert_the_real_reason_for_brains (consultado el 12 de febrero de 2014).

Zanuso, S., Sieverdes, J. C., Smith, N., Carraro, A., y Bergamin, M. (2012). The effect of a strength training program on affect, mood, anxiety, and strength performance in older individuals. International Journal of Sport Psychology, 43, 53-66.

Zeidner, M., Matthews, G., y Roberts, R. D. (2012). The Emotional Intelligence, Health, and Well-Being Nexus: What Have We Learned and What Have We Missed? Applied Psychology: Health and Well-Being, 1, 1-30. 